\title{
Radial-Velocity Standard Stars
}

\author{
Robert P. Stefanik, David W. Latham, and Guillermo Torres \\ Harvard-Smithsonian Center for Astrophysics, 60 Garden Street, \\ Cambridge, Massachusetts, 02138
}

\begin{abstract}
We review the history of the IAU Radial Velocity Standard Stars and give a status report on recent efforts at the HarvardSmithsonian Center for Astrophysics to establish an absolute velocity zero point for these stars and to improve their usefulness for intercomparing the results from different instruments and observatories.
\end{abstract}

\section{Introduction}

Radial velocity standard stars were originally proposed for two main purposes:

1. Intercomparisons between instruments. Stars designated as standards were meant to be well-suited for intercomparing results from different instruments and observatories. This purpose implied several desirable characteristics for sets of standard stars:

- They should be located near the celestial equator and distributed evenly around the sky in right ascension so that they are accessible to observatories located in both hemispheres at any time of the year or night.

- They should be well-established as constant stars so that variability does not have to be taken into account.

- Important parameters such as spectra type, velocity, and apparent brightness should be well covered.

2. Absolute velocity zero point. The absolute velocity zero point of the system should be well established, so that observations of the standards can be used to calibrate instrumental zero points.

Radial velocities of bright stars were being mass produced already in the early 1900s. However, recent improvements in the instruments and techniques used to measure radial velocities have put new demands on the performance expected from standard stars. In this paper we summarize the history of the IAU Radial Velocity Standard Stars, and give a status report on recent efforts at the Harvard-Smithsonian Center for Astrophysics (CfA) to improve the performance of the standards. We confine our discussion to stars with spectral types later than F5. 


\section{History of the IAU Standards}

The stars in the current "official" list of IAU Radial Velocity Standard Stars were drawn from three sources. There is also a fourth list (Evans 1967) of southern-hemisphere stars that we will not consider here.

1. Bright Standards (Pearce 1957). 25 stars observed at 14 different observatories; reduced to the Lick velocity system; brighter than $V$ magnitude 4.3; spectral type F0 and later; dwarfs and giants; number of observations per star: 19 to 528 .

2. Faint Standards (Pearce 1957). 35 stars observed at 7 different observatories; fainter than $V$ magnitude 4.3 and brighter than 8.0 ; spectral type F0 and later; dwarfs and giants; number of observations per star: 7 to 38 .

3. Heard-Fehrenbach Standards (Evans 1967, Bouigue 1973). 21 stars observed at the David Dunlap Observatory, the Observatoire de Haute Provence and the Dominion Astrophysical Observatory; $V$ magnitude 7 to 9 (the magnitudes were originally given as 8.2 to 9.7 , but these are off by about one magnitude); spectral types $\mathrm{F}$ to $\mathrm{K}$; dwarfs and giants; number of observations per star: 7 to 18 .

These 81 stars are the "official" list of IAU Radial Velocity Standard Stars. They are a smorgasbord of spectral types and luminosity classes, with velocities drawn from several observatories, using different techniques and dispersions, and covering various time spans and numbers of observations. It is not surprising that a list derived in this manner would present difficulties, particularly as modern velocity methods covering very long time spans are applied to new determinations of their velocities. Many have proven to be velocity variables.

To appreciate some of the difficulties associated with the official standards, it is helpful to review briefly some history (see also Batten 1978, 1985; Batten et al. 1983).

Shortly after the above stars were proposed as standards there were reports that several of the stars, particularly among the brighter giants, showed velocity variations larger than $1 \mathrm{~km} \mathrm{~s}^{-1}$. For example, HD 20902 and HD 45348 from the Bright list were rejected as standards in the 1958 IAU Commission 30 report to the General Assembly (Heard 1960). Other stars from the Bright and Faint lists were suspected of being variables and were recommended to be deleted from the standard list. Some of these have indeed been shown to be variable, but others have not been confirmed as variables.

The Heard-Fehrenbach list was derived from a survey of 1041 late-type stars started by F. Hogg and completed by J. Heard at the David Dunlap Observatory (Heard 1956). Twenty-four stars from that survey were originally proposed as standards by Heard as reported by Evans (1967). The 24 stars in the Evans list were subsequently observed by Heard and Ch. Fehrenbach at the David Dunlap, Haute Provence and Dominion Astrophysical Observatories (Heard \& Fehrenbach 1972). Three stars were found to be variable, and ultimately orbital solutions were obtained for all three: HD 160952, $P=182$ days and $K=2.64 \mathrm{~km}$ $\mathrm{s}^{-1}$ (Radford \& Griffin 1976); BD $+29^{\text {circ }} 1553, P=892$ days and $K=4.72 \mathrm{~km}$ $\mathrm{s}^{-1}$ (Stefanik, private communication); HD 204934, $P=144$ days and $K=5.90$ $\mathrm{km} \mathrm{s}^{-1}$ (Radford \& Griffin 1975, Bassett 1978). The remaining 21 stars were adopted as IAU standards as reported by Bouigue (1973). The adopted velocities 
were arrived at by shifting the velocities from each observatory to the IAU system defined by the Bright and Faint stars and averaging.

Thirteen of the Heard-Fehrenbach stars were observed by Griffin (1975), who found an additional variable star, HD 14969, which he later showed to be a spectroscopic binary (Griffin 1980). Griffin combined his observations with those of Heard \& Fehrenbach (1972), weighted by their respective errors, to suggest an "improved" list of velocities reduced to the IAU system. Griffin $(1969,1975)$ also criticized the method being used to establish a velocity system and adopted four of his own velocity standards, often referred to as the Griffin Standards.

Clear evidence for velocity variation among several more of the IAU standards was slowly accumulating, and the introduction of modern spectroscopic techniques promised to reveal additional problems. This was indeed the case. The first modern update of the IAU standards was presented at IAU Colloquium No. 88 by Mayor \& Maurice (1985) based on CORAVEL observations. They reported that the IAU velocities for the Bright and Faint standards were on different systems with a zero-point shift of $0.8 \mathrm{~km} \mathrm{~s}^{-1}$, and they noted that four of the IAU standards were variable, and three were possible variables. An orbit for one of the possible variables, HD 114762, was published in 1989 by Latham et al., with $P=84$ days and $K=0.59 \mathrm{~km} \mathrm{~s}^{-1}$. In 1990 Scarfe, Batten, \& Fletcher published an update of the velocities for the IAU standards based on observations made at the Dominion Astrophysical Observatory. It was clear that the IAU standards still included velocity variables, and a comparison of the results from different observatories showed systematic trends both with stellar color and velocity.

Because of these problems IAU Commission 30 formally addressed the issues of variability among the IAU standards and the zero-point of the IAU velocity system. It set the objective of establishing a new set of late-type IAU Radial Velocity Standard Stars with individual mean velocities and an absolute zero point of the entire system good to $100 \mathrm{~m} \mathrm{~s}^{-1}$. An observational campaign to monitor the IAU standards from several observatories was undertaken, including efforts with the Dominion Astrophysical Observatory (Victoria) spectrometer, the CORAVELs, and the CfA Digital Speedometers.

The status of this effort was summarized in the report of Commission 30 to the 1990 General Assembly (Latham \& Stefanik 1991). In that report the results from the three observatories along with the official IAU velocities were reported for 72 of the IAU standards. Removed from that summary, in addition to the three variables mentioned above, were stars found to be variable with a semi-amplitude larger than $1 \mathrm{~km} \mathrm{~s}^{-1}$ or whose IAU velocity appeared to be in error by more than $1 \mathrm{~km} \mathrm{~s}^{-1}$ : HD 36673,156014,35410, 44131, and 115521. Also removed was HD 184467, which McClure (1983) had shown to be a spectroscopic binary. Even with these removals, a number of suspected variables remained. And, a systematic color dependence between the velocities from the CORAVELS compared to Victoria and CfA was clearly evident.

At the 1988 General Assembly it had been agreed that an effort should be made to establish some additional $\mathrm{G}$ dwarfs as standards. The primary argument for concentrating on $\mathrm{G}$ dwarfs was that any absolute velocity zero point that was established by observing minor planets would most safely transfer to stars with essentially the same spectrum as the sun. The hope was that the 
impact of the color problem could be minimized this way. Indeed, comparisons between CfA and the CORAVELS gave the smallest differences for $G$ dwarfs. Furthermore, good candidates could be drawn from the samples of $G$ dwarfs that had been monitored for many years. The status of the results for 25 new G dwarf candidates was reported by Latham \& Stefanik (1991). Included were 18 stars chosen from the sample of $G$ dwarfs monitored with the CORAVELS (Duquennoy \& Mayor 1991) and 7 from the sample observed using a hydrogenfluoride gas absorption cell on the Canada-France-Hawaii Telescope (Campbell, Walker, \& Yang 1988; Walker et al. 1989). These G dwarf candidates all had a large number of observations covering long time spans, and showed no velocity variations. All appear to be good candidates for adoption as official standards, although it should be pointed out that subsequently a spectroscopic orbit with $K=0.06 \mathrm{~km} \mathrm{~s}^{-1}$ was published for one of them, HD $217014=51 \mathrm{Peg}$ (Mayor \& Queloz 1995)

\section{Comparison of the CfA and Victoria Results}

As a step towards improving the performance of the IAU standards, a careful comparison was made between the CfA and Victoria results during a visit of Scarfe to the CfA in 1993. A total of more than 9100 observations were compared, 6442 from the CfA Digital Speedometers, 1058 made by Scarfe with the DAO spectrometer, and 1606 made by McClure with the same instrument. These velocities have a typical precision of 0.4 or $0.5 \mathrm{~km} \mathrm{~s}^{-1}$ and covered a span of 10 to 15 years. The differences between the Victoria and CfA results showed no trends with mean velocity, right ascension, declination, magnitude, or spectral type.

This comparison disclosed clear evidence for velocity variations in four stars. HD 156014 and 115521 were confirmed as low-amplitude variables. HD 115521 shows a long-term variation with a timescale longer than 6000 days, and also a short term oscillation with period of about 470 days. HD 140913 gave a spectroscopic orbit with low amplitude (Stefanik et al. 1994; Mazeh, Latham, \& Stefanik 1996), and HD 171232 showed a decrease of $5 \mathrm{~km} \mathrm{~s}^{-1}$ over a period of 16 years. There was also a hint that HD 29587 was variable, and eventually a low-amplitude spectroscopic orbit was published for this star (Mazeh et al. 1996).

Two additional variables are HD 123782 , which has a semi-amplitude of $0.95 \mathrm{~km} \mathrm{~s}^{-1}$ and period of 493 days, and HD 42397, which was discovered by Scarfe (1992) to be a double-lined spectroscopic binary with long period and high eccentricity. This has been confirmed by additional CfA observations.

\section{Status of the CfA Effort}

For more than 15 years there has been an active program at the CfA to monitor the velocities of standard stars using the CfA Digital Speedometers (Latham 1985, 1992) on the 1.5-m Wyeth Reflector at the Oak Ridge Observatory located in the town of Harvard, Massachusetts, and on the 1.5-m Tillinghast Reflector and MMT, both located at the F. L. Whipple Observatory atop Mt. Hopkins, Arizona. 
1. Templates. For the first several years of operation of the CfA Digital Speedometers we used observed spectra as the templates for our cross-correlation velocity reduction procedures. Most of the time we used an observed spectrum of the dusk sky as the template, but we also used observed spectra of an A star or an M star for extreme cases. For most of the 1990s we used templates drawn from an extensive library of synthetic spectra calculated by Jon Morse using Kurucz model atmospheres (e.g. Nordström et al. 1994). A new and improved library of synthetic spectra has recently become available (Morse \& Kurucz in preparation), and we have been using these for templates since 1997.

2. Run-to-Run Velocity Shifts. We monitor the velocity zero point of the CfA Digital Speedometers using exposures of the dawn and dusk sky every night we observe. Usually these exposures show that it is sufficient to use a single correction for the zero point during an entire month's run, although occasionally there are significant shifts during a run due to changes in the instrument such as swapping detector packages. During the first few years of operation we did not monitor the dawn and dusk sky. To bring observations from those years onto the CfA sky-calibrated system we have solved for the run-to-run shifts using a global solution of 23572 observations of 1002 stars that have been observed since the beginning, including many IAU standards and stars in various binary surveys.

3. Absolute Velocity Zero Point. To establish the absolute velocity zero point of the CfA system and as an independent check of the long-term stability achieved using sky exposures for the run-to-run corrections, we have been monitoring minor planets for more than 13 years and have accumulated 1245 exposures of 35 different minor planets. The observed velocities are compared to velocities predicted from the astrometric orbit by the IAU Minor Planet Center (Marsden \& Bardwell, private communication). These observations confirm that there is no drift in the velocity zero point based on the sky exposures, but the sky calibration gives velocities which are too positive by $81 \mathrm{~m} \mathrm{~s}^{-1}$ when the old templates are used and by $136 \mathrm{~m} \mathrm{~s}^{-1}$ when the new templates are used (because we have chosen to continue to use the same old synthetic template for the sky velocity reductions, and there is a shift of $55 \mathrm{~m} \mathrm{~s}^{-1}$ between the old and new synthetic template for the sun). The formal uncertainty (the standard deviation of the mean) in these velocity shifts is $14 \mathrm{~m} \mathrm{~s}^{-1}$, but undoubtedly the systematic errors are larger. In particular, the gravitational redshift is not included in our synthetic spectra, so the CfA velocities for giants are systematically blueshifted by values on the order of $0.2 \mathrm{~km} \mathrm{~s}^{-1}$.

4. Standard Star Results. The status of the CfA observations of IAU standards and new $G$ dwarf candidate standards are summarized in Tables 1 and 2 , respectively. These velocities were derived using the new synthetic templates, so $136 \mathrm{~m} \mathrm{~s}^{-1}$ should be subtracted in order to transfer to the absolute velocity zero point established by the CfA observations of minor planets. Columns 1 to 3 give the star identifications; columns 4 and 5 the J2000 coordinates; column 8 and 9 the number of observations and the time spanned in days; column 10 the mean velocity; columns 11 and 12 the standard deviation of the mean and the standard deviation of an individual observation from the mean; column 13 the source of the star; and column 14 a recommendation for stars that should 
be rejected. Notes on the stars that are recommended for rejection are given below.

HD 14969. Griffin (1980) reported a spectroscopic orbit with $P=1935$ days and $K=4.43 \mathrm{~km} \mathrm{~s}^{-1}$.

HD 20902. Shortly after being proposed as a standard, this star was reported to have velocity variations larger than $2 \mathrm{~km} \mathrm{~s}^{-1}$ and was rejected (Heard $1960)$.

HD 29587. Mazeh et al. (1996) reported a spectroscopic orbit. A new CfA solution, updated to include the recent observations, gives $P=1470$ days and $K=0.89 \mathrm{~km} \mathrm{~s}^{-1}$.

HD 35410. The combined CfA and Victoria velocities give a spectroscopic orbit with $P=1493$ days, $K=1.96 \mathrm{~km} \mathrm{~s}^{-1}$, and $e=0.72$.

HD 36673 . The CORAVEL team reported this star to have velocity variations larger than $1 \mathrm{~km} \mathrm{~s}^{-1}$.

HD 42397. Scarfe (1992) reported this star to be a double-lined spectroscopic binary with long period and high eccentricity. Recent CfA observations confirm that the spectrum is composite.

HD 44131. Duquennoy \& Mayor (1991) reported a spectroscopic orbit with $P=3393$ days and $K=1.18 \mathrm{~km} \mathrm{~s}^{-1}$.

HD 45348. Shortly after being proposed as a standard, this star was reported to have velocity variations larger than $3 \mathrm{~km} \mathrm{~s}^{-1}$ and was rejected (Heard 1960).

HD 114762. Latham et al. (1989) reported a spectroscopic orbit with $P=84$ days and $K=0.59 \mathrm{~km} \mathrm{~s}^{-1}$. Mazeh et al. (1996) updated the orbital solution.

HD 115521. Many observers have reported that the velocity of this star is variable. There is a long-term variation, probably due to orbital motion with $P>6000$ days and $K \sim 4 \mathrm{~km} \mathrm{~s}^{-1}$. There is also a short-period oscillation with $P=470$ days.

HD 123782. Duquennoy \& Mayor (1991) reported a spectroscopic orbit with $P=494$ days and $K=0.87 \mathrm{~km} \mathrm{~s}^{-1}$.

HD 140913. Stefanik et al. (1994) reported a spectroscopic orbit based on the combined CfA and Victoria velocities. Mazeh et al. (1996) reported an updated orbital solution with $P=148$ days and $K=1.93 \mathrm{~km} \mathrm{~s}^{-1}$.

HD $156014=\alpha$ Her. This supergiant is a member of a quadruple system surrounded by a circumstellar envelope. The visual companion is itself a doublelined spectroscopic binary with $P=51.6$ days, and is separated by $4.7^{\prime \prime}$ from the primary. The primary itself has a speckle companion at a separation of $0.19^{\prime \prime}$ and expected period longer than $100 \mathrm{yr}$. The velocity variations of the primary exceed $1 \mathrm{~km} \mathrm{~s}^{-1}$, but show no clear periodicity.

HD 171232. The combined CfA and Victoria velocities show a slow drift downward of $5 \mathrm{~km} \mathrm{~s}^{-1}$ over the past $16 \mathrm{yr}$.

HD 184467. McClure (1983) reported that this star is a double-lined spectroscopic binary. CfA observations yield an orbit with $P=493$ days and $K=7$ $\mathrm{km} \mathrm{s}^{-1}$.

HD 206778. The CfA velocities show a recent decrease of about $1.5 \mathrm{~km} \mathrm{~s}^{-1}$.

HD 223094. The CfA velocities show a variation with $P \sim 500$ days and $K \sim 0.6 \mathrm{~km} \mathrm{~s}^{-1}$. 


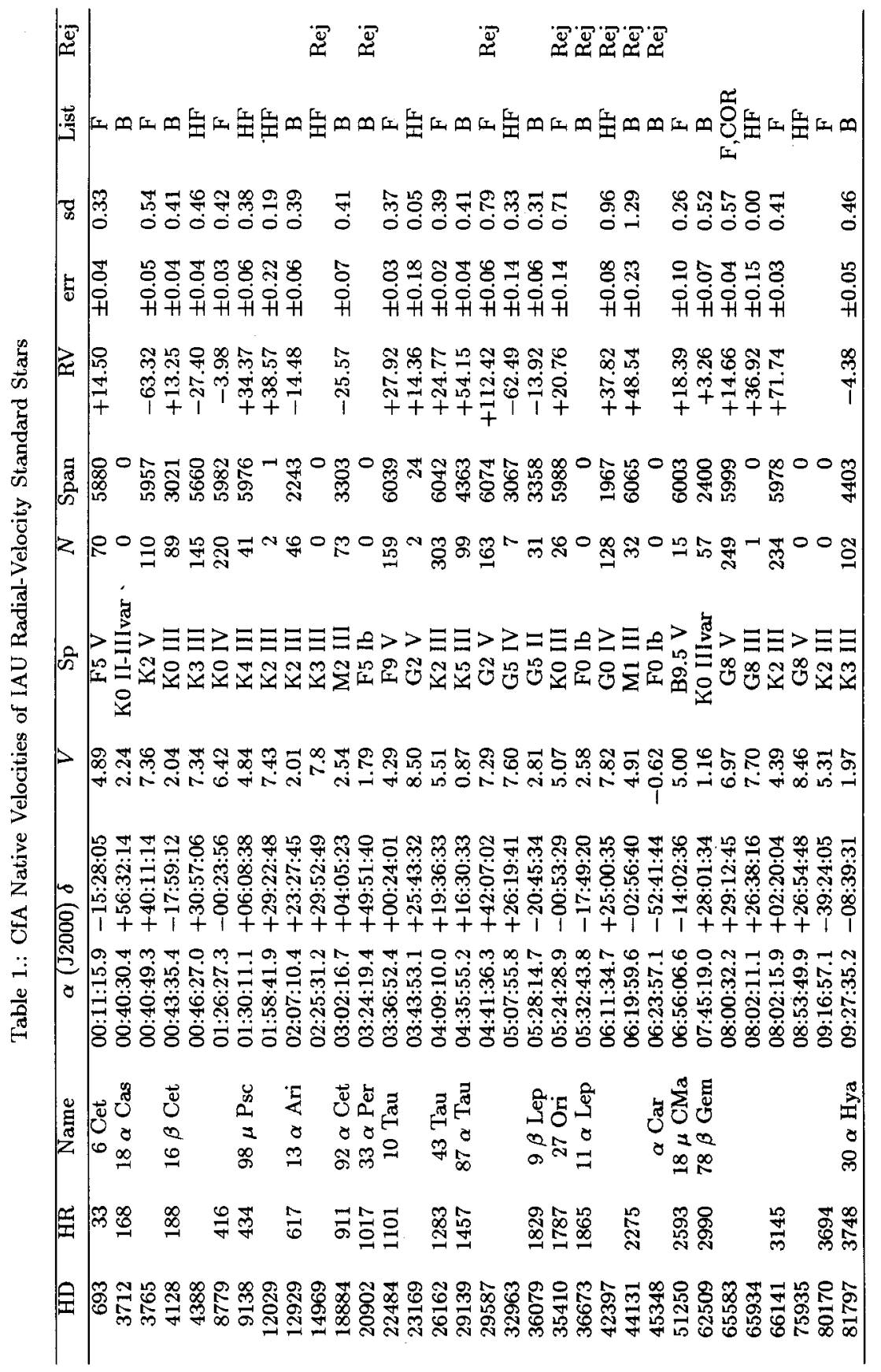




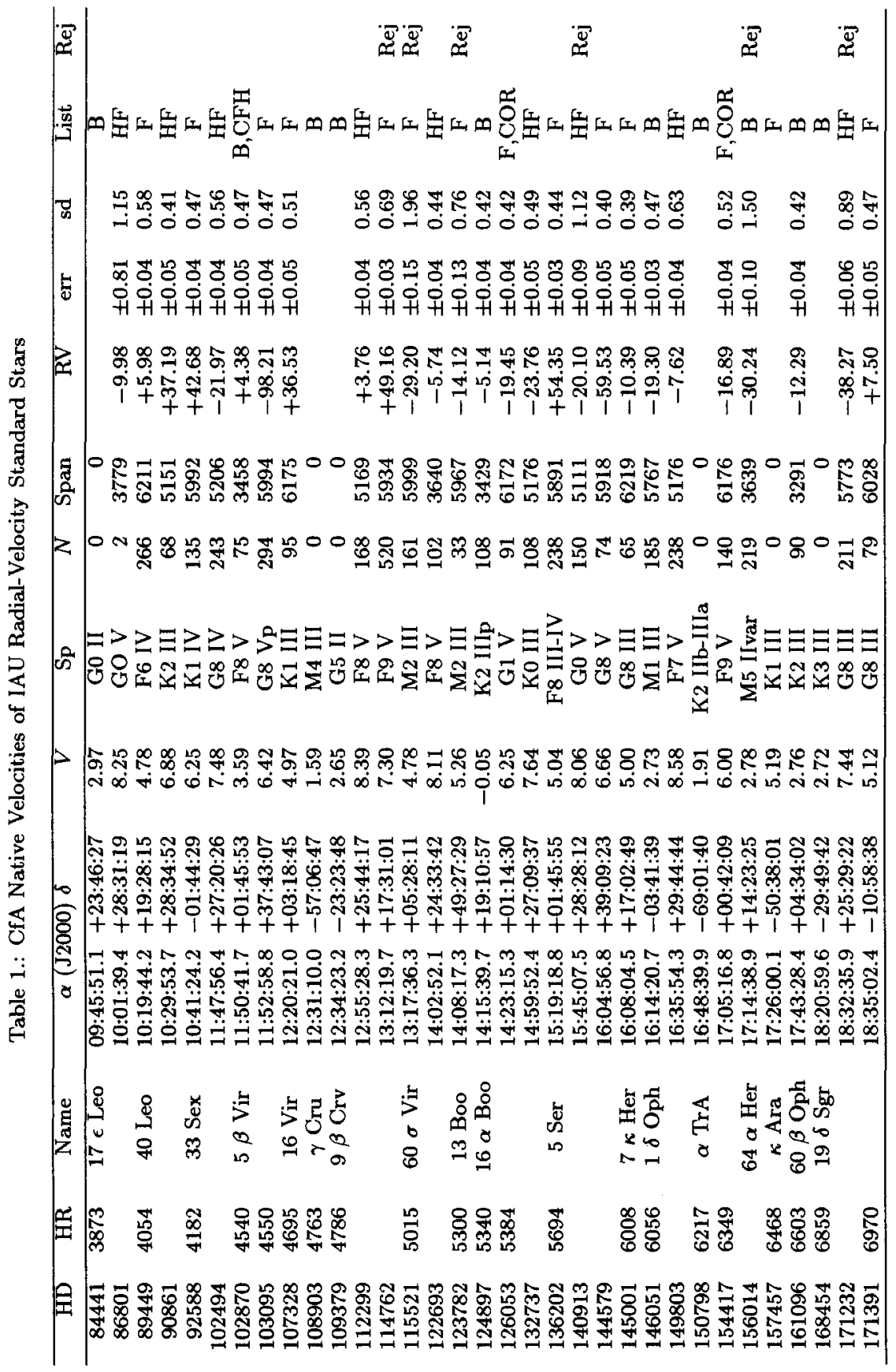




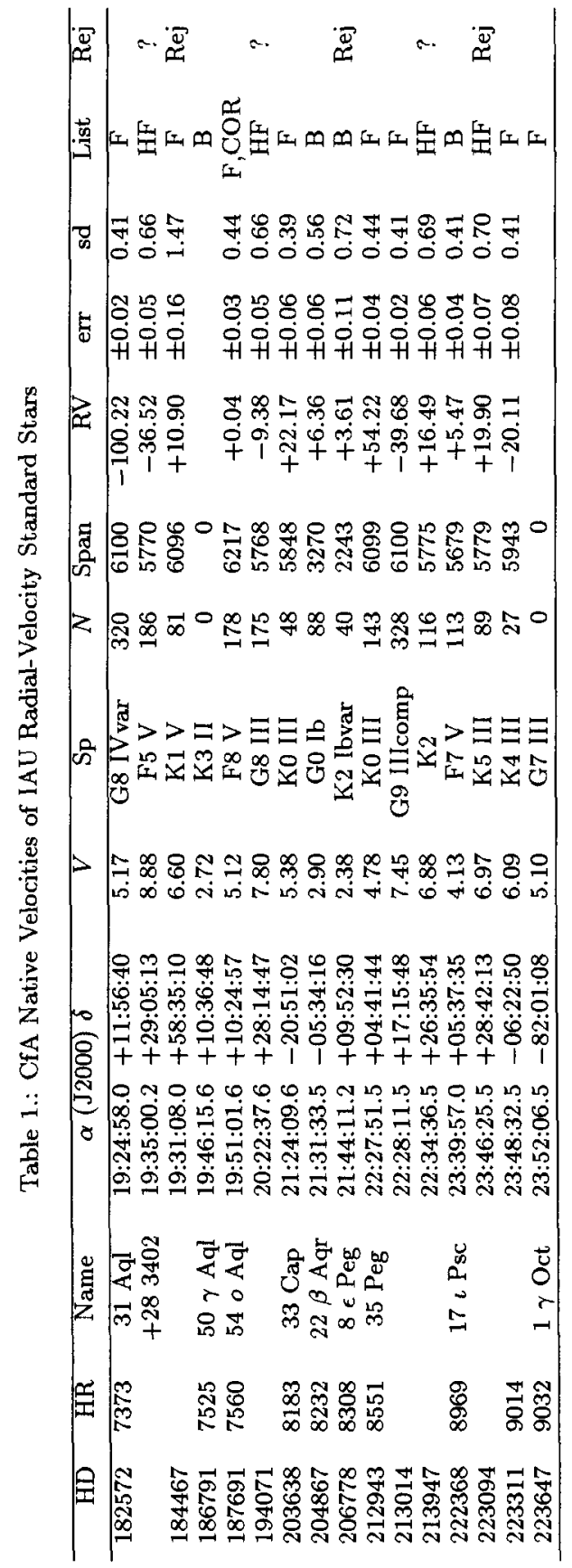




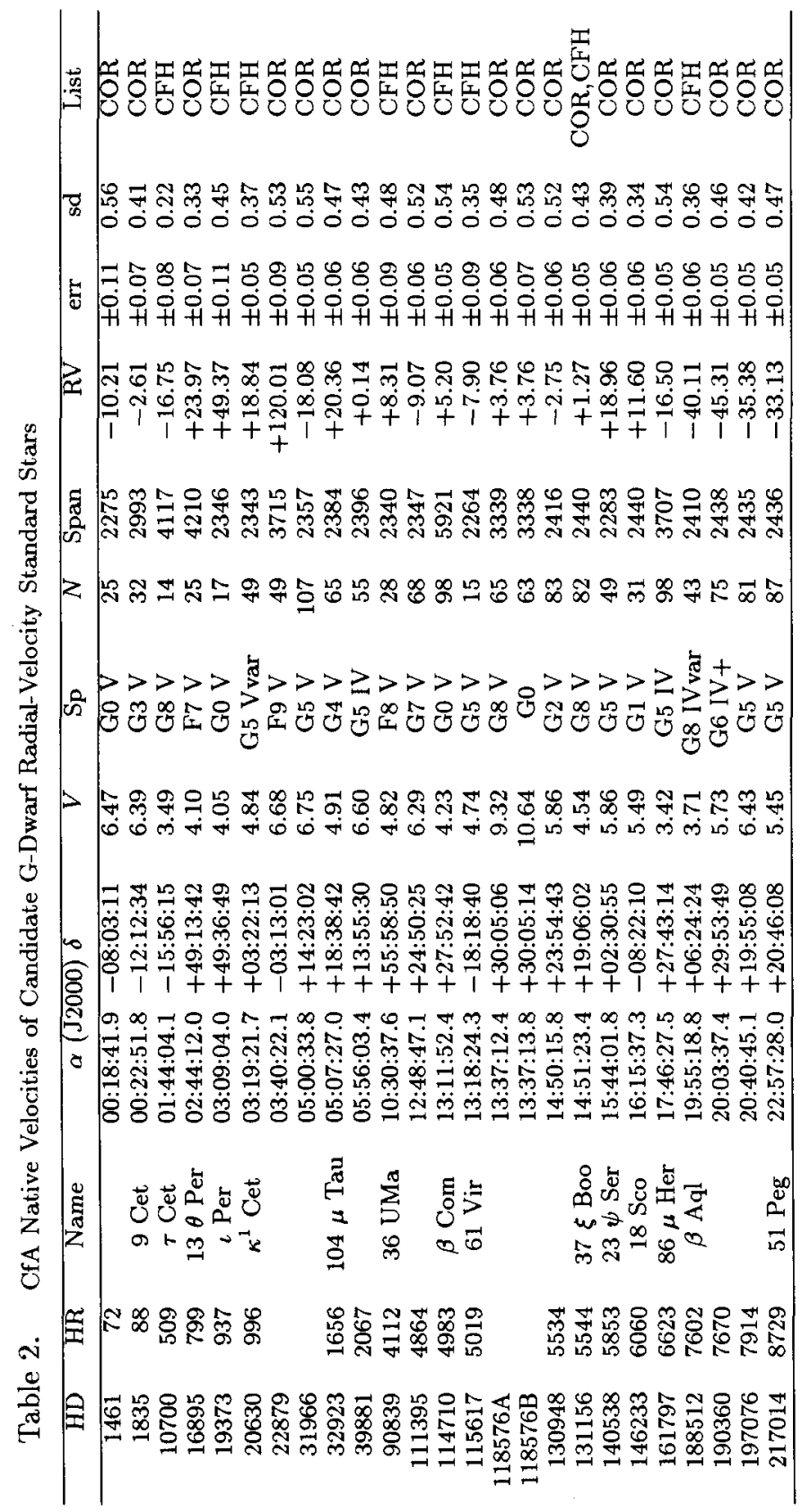




\section{References}

Batten, A.H. 1978, Vistas in Astronomy, 22, 265

Batten, A.H. 1985, in Stellar Radial Velocities (IAU Coll. 88), A.G.D. Philip \& D.W. Latham, Schenectady: L. Davis, 325

Batten, A.H., Harris, H., McClure, R.D., \& Scarfe, C.D. 1983, Publ. Dom. Astrophys. Obs., 16, 143

Bouigue, R. 1973, Trans. IAU, 15A, 407

Campbell, B., Walker, G.A.H., \& Yang, S. 1988, ApJ, 331, 902

Duquennoy, A., \& Mayor, M. 1991, A\&A, 248, 485

Bassett, E.E. 1978, Observatory, 98, 122

Evans, D.S. 1967, Trans. IAU, 13B, 170

Griffin, R.F. 1969, MNRAS, 145, 163

Griffin, R.F. 1975, MNRAS, 171, 407

Griffin, R.F. 1980, MNRAS, 190, 711

Heard, J.F. 1956, Publ. David Dunlap Obs., 2, 107

Heard, J.F. 1960, Trans. IAU, 10, 483

Heard, J.F., \& Fehrenbach, Ch. 1972, Publ. David Dunlap Obs., 3, 113

Latham, D.W. 1985, in Stellar Radial Velocities (IAU Coll. 88), A.G.D. Philip \& D.W. Latham, Schenectady: L. Davis, 21

Latham, D.W. 1992, in Complementary Approaches to Binary and Multiple Star Research (IAU Coll. 135), H.A. McAlister \& W.I. Hartkopf, San Francisco: Astron. Soc. Pacific, 32, 110

Latham, D.W., \& Stefanik, R.P. 1991, Trans. IAU, 21B, 269

Latham, D.W., Stefanik, R.P., Mazeh, T., Mayor, M., \& Burki, G. 1989, Nature, 339,38

Mayor, M., \& Maurice, E. 1985, in Stellar Radial Velocities (IAU Coll. 88), A.G.D. Philip \& D.W. Latham, Schenectady: L. Davis, 299

Mayor, M., \& Queloz, D. 1995, Nature, 378, 355

Mazeh, T., Latham, D.W., \& Stefanik, R.P. 1996, ApJ, 466, 415

McClure, R.D. 1983, PASP, 95, 201

Nordström, B., Latham, D.W., Morse, J.A., Kurucz, R.L., Andersen, J., \& Stefanik, R.P. 1994, A\&A, 287, 338

Pearce, J.A. 1957, Trans. IAU, 9, 441

Radford, G.A., \& Griffin, R.F. 1975, Observatory, 95, 187

Radford, G.A., \& Griffin, R.F. 1976, Observatory, 96, 56

Scarfe, C.D. 1992, Inf. Bull. Var. Stars, 3736

Scarfe, C.D., Batten, A.H., \& Fletcher, J.M. 1990, Publ. Dom. Astrophys. Obs., 18,21

Stefanik, R.P., Latham, D.W., Scarfe, C.D., Mazeh, T., Davis, R.J., \& Torres, G. 1994, BAAS, 184, 4307

Walker, G.A.H., Yang, S., Campbell, B., \& Irwin, A.W. 1989, ApJ, 343, L21 


\section{Discussion}

Soderblom: Someone should put up-to-date information on standards on a web page, for access by observers atthe telescope.

Stefanik: We do plan to put it all on the IAU Commission 30 web page.

Soderblom: This is something that could occupy you forever! How do you decide on what grounds to drop objects from your list?

Stefanik: We've pretty well dropped the giants, although Willie Torres continues to take an interest in them. For solar-type stars, we've done essentially all we can; we don't expect to find any more low-mass companions.

Hearnshaw: Why do we need standard stars anyway? Most work on precise velocities of variable stars is now differential. For studies of galactic dynamics we do want absolute velocities, but instead of using standard stars, I suspect that synthetic spectra provide a better standard, as the velocity is preceisely known, and complications such as convective shifts are absent.

Stefanik: One reason is to reduce data runs, by different observers over a long period of time, to a common system. It is also desirable to have stars known to be constant, so that people searching for vary small variations in stars can check the stability of their instruments. But I do agree that the future of standard stars is not clearcut.

Udry: Concerning the systematic color effect between CfA and Coravel, you use different synthetic spectra for different stars. Are you not risking the introduction of a color effect?

Stefanik: Comparisons with others, such as Griffin, for groups such as the Hyades, show no color effect. We try to match templates to the observed stars carefully, to avoid one. The amount that remains between Coravel and CfA is still a problem.

Latham: I have experimented with our library of synthetic templates, to see what kind of velocity errors can result from template mismatch. It is not difficult to get differences as large as $1 \mathrm{~km} \mathrm{~s}^{-1}$ from that source, when the templates are drawn from the range of temperatures covered by our library, 3500 to $11000 \mathrm{~K}$. Rotational velocity mismatch can be even more important, especially for large $v \sin i$ values, say in the range 50 to $100 \mathrm{~km} \mathrm{~s}^{-1}$. Our goal is to minimize the effects of mismatch by identifying a template that is closely similar to the observed spectrum, but it is hard to quantify how large the residual effects might be. I would be disappointed if they prove to be as large as $0.5 \mathrm{~km} \mathrm{~s}^{-1}$ for the cool dwarfs, as would be required to match the new proposed ELODIE velocity scale.

Gray: My comment is the flip side of John's. It seems to me that a coherent grid of radial velocities across the HR diagram is highly desirable, and if that is true, then we should be establishing what you call the absolute zero-point for all spectral types. Pinning the zero-point with the solar system is good, but not enough. Convective shifts of spectral lines vary systematically across the HR diagram, possibly from star to star, and certainly with wavelength. Do we have enough data in the world to look at things like the mean radial velocity of all 
B stars, or all A stars, for example, to see if there is a net residual? Do they appear to be expanding away from us or contracting toward us? Or have we looked at cluster stars to see if we get the same velocity from groups of stars along the main sequence? There may be other such tests. Although this is probably beyond the mandate of your original project, shouldn't someone be tackling this basic problem.

Stefanik: I think that's for the future. The early standards are still in difficult shape, as Frank Fekel will soon tell us. We're stuck with the current set of stars for historical reasons; they represent an enormous amount of work, which we don't want to throw out.

Hearnshaw: In stellar photometry, however, we junked the whole sysem in the 1950's and started again.

Stefanik: We haven't done that! 